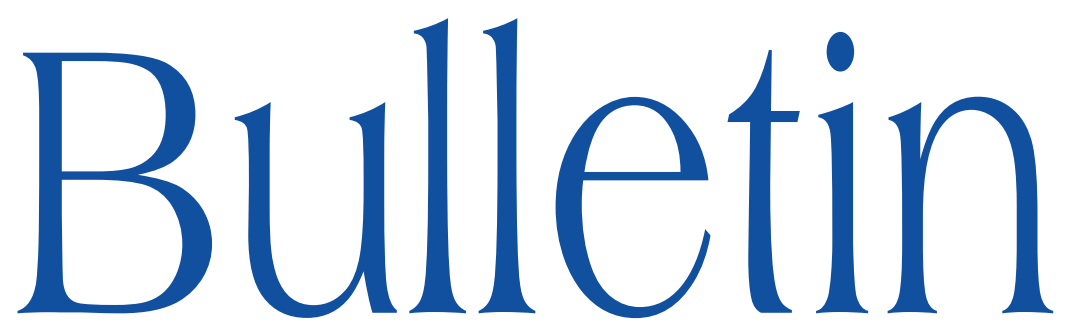

de la SOCIÉTÉ MATHÉMATIQUE DE FRANCE

\title{
RATIONAL BV-ALGEBRA IN STRING TOPOLOGY
}

\section{Yves Félix \& Jean-Claude Thomas}

\section{Tome 136}

Fascicule 2

2008 


\title{
RATIONAL BV-ALGEBRA IN STRING TOPOLOGY
}

\author{
By Yves Félix \& Jean-Claude Thomas
}

To Micheline Vigué-Poirrier on her 60th birthday

\begin{abstract}
Let $M$ be a 1-connected closed manifold of dimension $m$ and $L M$ be the space of free loops on $M$. M. Chas and D. Sullivan defined a structure of BValgebra on the singular homology of $L M, H_{*}(L M ; \boldsymbol{k})$. When the ring of coefficients is a field of characteristic zero, we prove that there exists a BV-algebra structure on the Hochschild cohomology $H H^{*}\left(C^{*}(M) ; C^{*}(M)\right)$ which extends the canonical structure of Gerstenhaber algebra. We construct then an isomorphism of BV-algebras between $H H^{*}\left(C^{*}(M) ; C^{*}(M)\right)$ and the shifted homology $H_{*+m}(L M ; \boldsymbol{k})$. We also prove that the Chas-Sullivan product and the BV-operator behave well with a Hodge decomposition of $H_{*}(L M)$.
\end{abstract}

Texte reçu le 7 juin 2007, accepté le 30 novembre 2007

Yves Félix, Université Catholique de Louvain, Département de mathématique, 2, Chemin du Cyclotron, 1348 Louvain-La-Neuve (Belgium) • E-mail : felix@math.ucl.ac.be Jean-Claude Thomas, Faculté des Sciences, LAREMA-UMR au CNRS 6093, 2, Bd. Lavoisier, 49045 Angers (France) • E-mail : jean-claude.thomas@univ-angers.fr 2000 Mathematics Subject Classification. — 55P35, 54N33, 81T30.

Key words and phrases. - String homology, rational homotopy, Hochschild cohomology, free loop space homology.

The authors are partially supported by INTAS program 03513251 . 
RÉSumé ( $B V$-algèbres rationnelles en topologie des lacets libres)

Soit $M$ une variété simplement connexe compacte sans bord de dimension $m$. Désignons par $L M$ l'espace des lacets libres sur $M$. M. Chas et D. Sullivan ont défini une structure de BV-algèbre sur l'homologie singulière $H_{*}(L M ; \boldsymbol{k})$. Lorsque l'anneau des coefficients $\boldsymbol{k}$ est un corps de caractéristique nulle, nous établissons l'existence d'une structure de BV-algèbre sur la cohomologie de Hochschild $H H^{*}\left(C^{*}(M) ; C^{*}(M)\right)$ qui étend la structure canonique d'algèbre de Gerstenhaber. De plus nous construisons un isomorphisme de BV-algèbres entre $H_{*+m}(L M ; \boldsymbol{k})$ et $H H^{*}\left(C^{*}(M) ; C^{*}(M)\right)$. Finalement nous démontrons que le produit de Chas-Sullivan ainsi que le BV-opérateur sont compatibles avec la décomposition de Hodge de $H_{*}(L M ; \boldsymbol{k})$.

\section{Introduction}

Chas and Sullivan considered in [3] the free loop space $L M=\operatorname{map}\left(S^{1}, M\right)$ for a smooth orientable closed manifold of dimension $m$. They use geometric methods to show that the shifted homology $\mathbb{H}_{*}(L M):=H_{*+m}(L M)$ has the structure of a Batalin-Vilkovisky algebra (BV-algebra for short). Later on Cohen and Jones defined in [5] a ring spectrum structure on the Thom spectrum $L M^{-T M}$ which realizes the Chas-Sullivan product in homology. More recently, Gruher and Salvatore proved in [17] that the algebra structure (and thus the BV-algebra structure) on $\mathbb{H}_{*}(L M)$ is natural with respect to smooth orientation preserving homotopy equivalences.

Assume that the coefficients ring is a field. By a result of Jones, [19, Thm. 4.1] there exists a natural linear isomorphism

$$
H H_{*}\left(C^{*}(M) ; C^{*}(M)\right) \cong H^{*}(L M),
$$

and by duality an isomorphism $H_{*}(L M) \cong H H^{*}\left(C^{*}(M) ; C_{*}(M)\right)$. Here $H H_{*}(A ; Q)$ (respectively $H H^{*}(A ; Q)$ ) denotes the Hochschild homology (respectively cohomology) of a differential graded algebra $A$ with coefficients in the differential graded $A$-bimodule $Q, C^{*}(M)$ denotes the singular cochains algebra and $C_{*}(M)$ the complex of singular chains. The cap product induces an isomorphism of graded vector spaces (for instance see [11, Appendix]), $H H^{*}\left(C^{*}(M) ; C_{*}(M)\right) \cong H H^{*-m}\left(C^{*}(M) ; C^{*}(M)\right)$, and therefore an isomorphism of graded vector spaces

$$
\mathbb{H}_{*}(L M) \cong H H^{*}\left(C^{*}(M) ; C^{*}(M)\right) .
$$

Since $H H^{*}(A ; A)$ is canonically a Gerstenhaber algebra, for any differential graded algebra $A$, it is natural to ask:

Question 1. - Does there exist an isomorphism of Gerstenhaber algebras between $\mathbb{H}_{*}(L M)$ and $H H^{*}\left(C^{*}(M) ; C^{*}(M)\right)$ ? 
Various isomorphisms of graded algebras have been constructed. The first one has been constructed by Merkulov for real coefficients [24], [13] using iterated integrals. An another isomorphism has been constructed for rational coefficients by M. Vigué and the two authors, [12], using the chain coalgebra of the Quillen minimal model of $M$.

Although $H H^{*}(A ; A)$ does not have, for any differential graded algebra $A$, a natural structure of BV-algebra extending the canonical Gerstenhaber algebra, a second natural question is:

Question 2. - Does there exist on $H H^{*}\left(C^{*}(M) ; C^{*}(M)\right)$ a structure of $B V$ algebra extending the structure of Gerstenhaber algebra and an isomorphism of $B V$-algebras between $\mathbb{H}_{*}(L M)$ and $H H^{*}\left(C^{*}(M) ; C^{*}(M)\right)$ ?

The main result of this paper furnishes a positive answer to Question 2 and thus to Question 1 when the field of coefficients is assumed of characteristic zero.

THEOREM 1. - If $M$ is 1-connected and the field of coefficients has characteristic zero then

(i) Poincaré duality induces a BV-structure on $H H^{*}\left(C^{*}(M) ; C^{*}(M)\right)$ extending the structure of Gerstenhaber algebra;

(ii) there exits an isomophism of BV-algebras

$$
\mathbb{H}_{*}(L M) \cong H H^{*}\left(C^{*}(M) ; C^{*}(M)\right) .
$$

$\mathrm{BV}$-algebra structures on the Hochschild cohomology $H H^{*}(A ; A)$ have been constructed by different authors under some conditions on $A$. First of all, Tradler and Zeinalian [29] did it when $A$ is the dual of an $A_{\infty}$-coalgebra with $\infty$ duality (rational coefficients). This is in particular the case when $A=C^{*}(M)$, see [28]. Menichi [23] constructed also a BV-structure in the case when $A$ is a symmetric algebra (any coefficients). Let us mention that Ginzburg [16, Thm. 3.4.3] has proved that $H H^{*}(A ; A)$ is a BV-algebra for certain algebras $A$. Using this result Vaintrob [30] constructed an isomorphism of BV-algebras between $\mathbb{H}_{*}(L M)$ and $H H^{*}(A ; A)$ when $A$ is the group ring with rational coefficients of the fundamental group of an aspherical manifold $M$. This is coherent with our Theorem 1 because in this case $C_{*}(\Omega M)$ is quasi-isomorphic to $A$ and using [9, Prop. 3.3] we have isomorphisms of Gerstenhaber algebras

$$
H H^{*}(A ; A) \cong H H^{*}\left(C_{*}(\Omega M) ; C_{*}(\Omega M)\right) \cong H H^{*}\left(C^{*}(M) ; C^{*}(M)\right) .
$$

Extending Theorem 1 to finite fields of coefficients would be difficult. For instance Menichi [22] proved that algebras $\mathbb{H}_{*}\left(L S^{2}\right)$ and $H H^{*}\left(H^{*}\left(S^{2}\right) ; H^{*}\left(S^{2}\right)\right)$ 
are isomorphic as Gerstenhaber algebras but not as BV-algebras for $\mathbb{Z} / 2$ coefficients.

In this paper we work over a field of characteristic zero. We use rational homotopy theory for which we refer systematically to [7]. We only recall here that a morphism in some category of complexes is a quasi-isomorphism if it induces an isomorphism in homology. Two objects are quasi-isomorphic if they are related by a finite sequence of quasi-isomorphisms. We shall use the classical convention $V^{i}=V_{-i}$ for degrees and $V^{\vee}$ denotes the graded dual of the graded vector space $V$.

Let $\boldsymbol{C}_{*}(A ; A):=(A \otimes T(s \bar{A}), \partial)$ be the Hochschild chain complex of a differential graded algebra $A$ with coefficients in $A$. Here $T(s \bar{A})$ denotes the free coalgebra generated by the graded vector space $s \bar{A}$ with $\bar{A}=\left\{A^{i}\right\}_{i \geq 1}$ and $(s \bar{A})^{i}=A^{i+1}$. We emphazise that $\boldsymbol{C}_{*}(A ; A)=A \otimes T(s \bar{A})$ is considered as a cochain complex for upper degrees.

Now by a recent result of Lambrechts and Stanley [20] there is a commutative differential graded algebra $A$ satisfying:

1) $A$ is quasi-isomorphic to the differential graded algebra $C^{*}(M)$.

2) $A$ is connected, finite dimensional and satisfies Poincaré duality in dimension $m$. This means there exists a $A$-linear isomorphism $\theta: A \rightarrow A^{\vee}$ of degree $-m$ which commutes with the differentials.

We call $A$ a Poincaré duality model for $M$.

The starting point of the proof is to replace $C^{*}(M)$ by $A$ because there is an isomorphism of Gerstenhaber algebras, [9, Prop. 3.3],

$$
H H^{*}(A ; A) \cong H H^{*}\left(C^{*}(M) ; C^{*}(M)\right) \text {. }
$$

This will allows us to use Poincaré duality at the chain level.

Denote by $\mu$ the multiplication of $A$. This is a model of the diagonal map. We define then the linear map $\mu_{A}: A \rightarrow A \otimes A$ by the commutative diagram

$$
\begin{aligned}
A^{\vee} \stackrel{\mu^{\vee}}{\longrightarrow}(A \otimes A)^{\vee} & =A^{\vee} \otimes A^{\vee} \\
\theta \uparrow & \cong \uparrow \theta \otimes \theta \\
A & \stackrel{\mu_{A} \longrightarrow A}{\longrightarrow} A \otimes A
\end{aligned}
$$

By definition $\mu_{A}$ is a $A \otimes A$-linear map degree $m$ which commutes with the differentials (Here $A$ is a $A \otimes A$-module via $\mu$ ). This is a representative of the Gysin map associated to the diagonal embedding. With these notation we prove in $\S 4$ : 
Proposition 1. - 1) The cochain complex $\boldsymbol{C}_{*}(A ; A)$ is quasi-isomorphic to the complex $C^{*}(L M)$. In particular, there is an isomorphism of graded vector spaces

$$
H H_{*}(A ; A) \cong H^{*}(L M) .
$$

2) If $\mu$ denotes the multiplication of $A$ and $\phi$ denotes the coproduct of the coalgebra $T(s \bar{A})$ then the composite $\Phi$

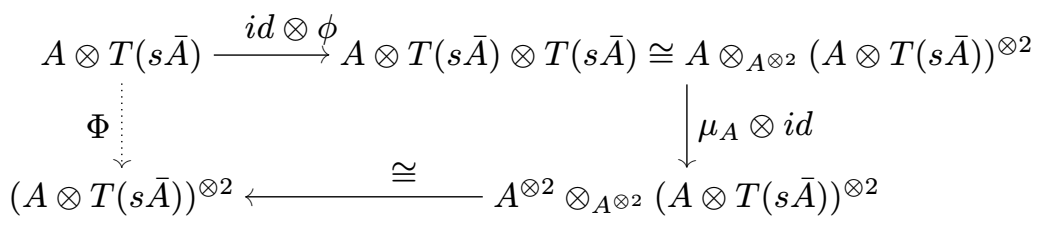

is a linear map of degree $m$ which commutes with the differentials.

3) The isomorphism $H H_{*}(A ; A) \cong H^{*}(L M)$, considered in 1$)$, transfers the map induced by $\Phi$ on $H_{*}(A ; A)$ to the dual of the Chas-Sullivan product on $H^{*-m}(L M)$.

4) The duality isomorphism $H_{*}(A ; A)^{\vee} \cong H H^{*}\left(A ; A^{\vee}\right) \stackrel{(\theta)}{\cong} H H^{*-m}(A ; A)$ transfers the map induced by $\Phi$ on $H_{*}(A ; A)^{\vee}$ to the Gerstenhaber product on $H H^{*}(A ; A)$.

Denote by $\Delta: \mathbb{H}_{*}(L M) \rightarrow \mathbb{H}_{*+1}(L M)$ and $\Delta^{\prime}: \mathbb{H}^{*}(L M) \rightarrow \mathbb{H}^{*-1}(L M)$ the morphisms induced by the canonical action of $S^{1}$ on $L M$. As proved by Chas and Sullivan this operator $\Delta$ defines on $\mathbb{H}_{*}(M)$ a structure of BV-algebra. In section 5 we prove:

Proposition 2. - The isomorphism $H_{*}(A ; A) \cong H^{*}(L M)$, considered in Proposition 1, transfers Connes' boundary $B: H H_{*}(A ; A) \rightarrow H H_{*+1}(A ; A)$ to the operator $\Delta^{\prime}$.

L. Menichi [23] proved that the duality isomorphism

$$
H H_{*}(A ; A)^{\vee} \cong H H^{*}\left(A ; A^{\vee}\right) \stackrel{(\theta)}{\cong} H H^{*}(A ; A)
$$

transfers $B^{\vee}:\left(H H_{*+1}(A ; A)^{\vee} \rightarrow\left(H H_{*}(A ; A)\right)^{\vee}\right.$ to a BV-operator on $H H^{*}(A ; A)$ that defines a $\mathrm{BV}$-structure extending the Gerstenhaber algebra structure. The isomorphisms of Gerstenhaber algebras (1) carries on the right hand term a structure of BV-algebra extending the Gerstenhaber algebra. This fact combined with Proposition 1 and 2 gives Theorem 1.

Since the field of coefficients is of characteristic zero, the homology of $L M$ admits a Hodge decomposition, $\mathbb{H}_{*}(L M)=\bigoplus_{r \geq 0} \mathbb{H}_{*}^{[r]}(L M)$ (see [33], [32], [15] 
and [21, Thm. 4.5.10]). We prove that this decomposition behaves well with respect to the product $\bullet$ and the BV-operator $\Delta$ defined by Chas-Sullivan.

THEOREM 2. - With the above notation, we have

1) $\mathbb{H}_{*}^{[r]}(L M) \otimes \mathbb{H}_{*}^{[s]}(L M) \stackrel{\bullet}{\longrightarrow} \mathbb{H}_{*}^{[\leq r+s]}(L M)$,

2) $\Delta: \mathbb{H}_{*}^{[r]}(L M) \longrightarrow \mathbb{H}_{*+1}^{[r+1]}(L M)$.

By definition $\mathbb{H}_{*}^{[0]}(L M)$ is the image of $H_{*+m}(M)$ by the homomorphism induced in homology by the canonical section $M \rightarrow L M$. It has been proved in [10] that if aut $M$ denotes the monoid of (unbased) self-equivalences of $M$ then there exists a natural isomorphism of graded algebras

$$
\mathbb{H}_{*}^{[1]}(L M) \cong H_{*+m}(M) \otimes \pi_{*}(\Omega \text { aut } M) .
$$

For any $r \geq 0$, a description of $\mathbb{H}_{*}^{[r]}(L M)$ can be obtained, using a Lie model $(L, d)$ of $M$, as proved in the last result.

Proposition 3. - The graded vector space $\mathbb{H}_{*}^{[r]}(L M)$ is isomorphic to $\operatorname{Tor}^{U L}\left(\boldsymbol{k}, \Gamma^{r}(L)\right)$ where $\Gamma^{r}(L)$ is the sub-UL-module of $U L$ for the adjoint representation that is the image of $\bigwedge^{r} L$ by the classical Poincaré-Birkoff-Witt isomorphism of coalgebras $\wedge L \rightarrow U L$.

The text is organized as follows. Notation and definitions are made precise in sections 2 and 3. Proposition 1 is proved in Sections 4, Proposition 2 is proved in section 5. Theorem 2 and Proposition 3 are proved in the last section.

\section{Hochschild homology and cohomology}

2.1. Bar construction. - Let $A$ be a differential graded augmented cochain algebra and let $P$ (res. $N$ ) be a differential graded right (resp. left) $A$-module,

$$
A=\left\{A^{i}\right\}_{i \geq 0}, \quad P=\left\{P^{j}\right\}_{j \in \mathbb{Z}}, \quad N=\left\{N^{j}\right\}_{j \in \mathbb{Z}} \quad \text { and } \quad \bar{A}=\operatorname{ker}(\varepsilon: A \rightarrow \boldsymbol{k}) .
$$

The two-sided (normalized) bar construction,

$$
\mathbb{B}(P ; A ; N)=P \otimes T(s \bar{A}) \otimes N, \quad \mathbb{B}_{k}(P ; A ; N)^{\ell}=\left(P \otimes T^{k}(s \bar{A}) \otimes N\right)^{\ell},
$$

is the cochain complex defined as follows. For $k \geq 1$, a generic element $p\left[a_{1}\left|a_{2}\right| \cdots \mid a_{k}\right] n$ in $\mathbb{B}_{k}(P ; A ; N)$ has (upper) degree $|p|+|n|+\sum_{i=1}^{k}\left(\left|s a_{i}\right|\right)$. If $k=0$, we write $p[] n=p \otimes 1 \otimes n \in P \otimes T^{0}(s \bar{A}) \otimes N$. The differential $d=d_{0}+d_{1}$ is defined by

$$
\mathbb{B}_{k}(P ; A ; N)^{\ell} \stackrel{d_{0}}{\longrightarrow} \mathbb{B}_{k}(P ; A ; N)^{\ell+1}
$$




$$
\begin{gathered}
\begin{array}{c}
d_{0}\left(p\left[a_{1}\left|a_{2}\right| \cdots \mid a_{k}\right] n\right)=d(p)\left[a_{1}\left|a_{2}\right| \cdots \mid a_{k}\right] n \\
-\sum_{i=1}^{k}(-1)^{\epsilon_{i}} p\left[a_{1}\left|a_{2}\right| \cdots\left|d\left(a_{i}\right)\right| \cdots \mid a_{k}\right] n \\
+(-1)^{\epsilon_{k+1}} p\left[a_{1}\left|a_{2}\right| \cdots \mid a_{k}\right] d(n), \\
\mathbb{B}_{k}(P ; A ; N)^{\ell} \stackrel{d_{1}}{\longrightarrow} \mathbb{B}_{k-1}(P ; A ; N)^{\ell+1}, \\
d_{1}\left(p\left[a_{1}\left|a_{2}\right| \cdots \mid a_{k}\right] n\right)=(-1)^{|p|} p a_{1}\left[a_{2}|\cdots| a_{k}\right] n \\
+\sum_{i=2}^{k}(-1)^{\epsilon_{i}} p\left[a_{1}\left|a_{2}\right| \cdots\left|a_{i-1} a_{i}\right| \cdots \mid a_{k}\right] n \\
-(-1)^{\epsilon_{k}} p\left[a_{1}\left|a_{2}\right| \cdots \mid a_{k-1}\right] a_{k} n .
\end{array}
\end{gathered}
$$

Here $\epsilon_{i}=|p|+\sum_{j<i}\left(\left|s a_{j}\right|\right)$.

In particular, considering $\boldsymbol{k}$ as a trivial $A$-bimodule we obtain the complex

$$
\mathbb{B} A=\mathbb{B}(\boldsymbol{k} ; A ; \boldsymbol{k})
$$

which is a differential graded coalgebra whose comultiplication is defined by

$$
\phi\left(\left[a_{1}|\cdots| a_{r}\right]\right)=\sum_{i=0}^{r}\left[a_{1}|\cdots| a_{i}\right] \otimes\left[a_{i+1}|\cdots| \mid a_{r}\right] .
$$

Recall that a differential $A$-module $N$ is called semifree if $N$ is the union of an increasing sequence of sub-modules $N(i), i \geq 0$, such that each $N(i) / N(i-1)$ is an $R$-free module on a basis of cycles (see [7]). Then,

Lemma 1 (see [7, Lemma 4.3]). - The canonical map $\varphi: \mathbb{B}(A ; A ; A) \rightarrow A$ defined by $\varphi[]=1$ and $\varphi\left(\left[a_{1}|\cdots| a_{k}\right]\right)=0$ if $k>0$, is a semifree resolution of $A$ as an $A$-bimodule.

2.2. Hochschild complexes. - Let us denote by $A^{e}=A \otimes A^{\text {op }}$ the envelopping algebra of $A$.

If $P$ is a differential graded right $A^{e}$-module then the cochain complex

$$
\boldsymbol{C}_{*}(P ; A):=(P \otimes T(s \bar{A}), \partial) \stackrel{\text { def }}{\cong} P \otimes_{A^{e}} \mathbb{B}(A ; A ; A),
$$

is called the Hochschild chain complex of $A$ with coefficients in $P$. Its homology is called the Hochschild homology of $A$ with coefficients in $P$ and is denoted by $H H_{*}(A ; P)$. When we consider $\boldsymbol{C}_{*}(A ; A)$ as well as $H H_{*}(A ; A), A$ is supposed equipped with its canonical right $A^{e}$-module structure.

For sake of completeness, let us recall the definition of the Connes' coboundary:

$$
B: \boldsymbol{C}_{*}(A ; A) \longrightarrow \boldsymbol{C}_{*}(A ; A) .
$$


One has $B\left(a_{0} \otimes\left[a_{1}|\cdots| a_{n}\right]\right)=0$ if $\left|a_{0}\right|=0$ and

$$
B\left(a_{0} \otimes\left[a_{1}|\cdots| a_{n}\right]\right)=\sum_{i=0}^{n}(-1)^{\bar{\epsilon}_{i}} 1 \otimes\left[a_{i}|\cdots| a_{n}\left|a_{0}\right| a_{1}|\cdots| a_{i-1}\right]
$$

if $\left|a_{0}\right|>0$, where

$$
\bar{\epsilon}_{i}=\left(\left|s a_{0}\right|+\left|s a_{1}\right|+\cdots+\left|s a_{i-1}\right|\right)\left(\left|s a_{i}\right|+\cdots+\left|s a_{n}\right|\right) .
$$

It is well known that $B^{2}=0$ and $B \circ \partial+\partial \circ B=0$. We also denote by $B$ the induced operator in Hochschild homology $H_{*}(A ; A)$.

If $N$ is a (left) differential graded $A^{e}$-module then the ( $\mathbb{Z}$-graded) complex

$$
C^{*}(A ; N):=(\operatorname{Hom}(T(s \bar{A}), N), \delta) \stackrel{\text { def }}{\cong} \operatorname{Hom}_{A^{e}}(\mathbb{B}(A ; A ; A), N)
$$

is called the Hochschild cochain complex of $A$ with coefficients in the differential graded $A$-bimodule $N$. Its homology is called the Hochschild cohomology of $A$ with coefficients in $N$ and is denoted by $H H^{*}(A ; N)$. When we consider $C^{*}(A ; A)$ as well as $H H^{*}(A ; A), A$ is supposed equipped with its canonical left $A^{e}$-bimodule structure.

Consider the graded dual, $V^{\vee}$, of the graded vector space $V=\left\{V^{i}\right\}_{i \in \mathbb{Z}}$, i.e. $V^{\vee}=\left\{V_{i}^{\vee}\right\}_{i \in \mathbb{Z}}$ with $V_{i}^{\vee}:=\operatorname{Hom}\left(V^{i}, \boldsymbol{k}\right)$. The canonical isomorphism

$$
\operatorname{Hom}\left(A \otimes_{A^{e}} \mathbb{B}(A ; A ; A), \boldsymbol{k}\right) \longrightarrow \operatorname{Hom}_{A^{e}}\left(\mathbb{B}(A ; A ; A), A^{\vee}\right)
$$

induces the isomorphism of complexes $\boldsymbol{C}_{*}(A ; A)^{\vee} \rightarrow \boldsymbol{C}^{*}\left(A ; A^{\vee}\right)$.

2.3. The Gerstenhaber algebra on $H H^{*}(A ; A)$. - A Gerstenhaber algebra is a commutative graded algebra $H=\left\{H_{i}\right\}_{i \in \mathbb{Z}}$ with a bracket

$$
H_{i} \otimes H_{j} \rightarrow H_{i+j+1}, \quad x \otimes y \mapsto\{x, y\}
$$

such that for $a, a^{\prime}, a^{\prime \prime} \in H$ :

(a) $\left\{a, a^{\prime}\right\}=(-1)^{(|a|-1)\left(\left|a^{\prime}\right|-1\right)}\left\{a^{\prime}, a\right\}$;

(b) $\left\{a,\left\{a^{\prime}, a^{\prime \prime}\right\}\right\}=\left\{\left\{a, a^{\prime}\right\}, a^{\prime \prime}\right\}+(-1)^{(|a|-1)\left(\left|a^{\prime}\right|-1\right)}\left\{a^{\prime},\left\{a, a^{\prime \prime}\right\}\right\}$.

For instance the Hochschild cohomology $H^{*}(A ; A)$ is a Gerstenhaber algebra [14]. The bracket can be defined by identifying $\boldsymbol{C}^{*}(A ; A)$ with a differential graded Lie algebra of coderivations (see [26] and [9, 2.4]).

TOME $136-2008-\mathrm{N}^{\mathrm{O}} 2$ 
2.4. BV-algebras and differential graded Poincaré duality algebras. - A BatalinVilkovisky algebra (BV-algebra for short) is a commutative graded algebra, $H$ together with a linear map (called a BV-operator)

$$
\Delta: H^{k} \longrightarrow H^{k-1}
$$

such that:

1) $\Delta \circ \Delta=0$

2) $H$ is a Gerstenhaber algebra with the bracket defined by

$$
\left\{a, a^{\prime}\right\}:=(-1)^{|a|}\left(\Delta\left(a a^{\prime}\right)-\Delta(a) a^{\prime}-(-1)^{|a|} a b \Delta\left(a^{\prime}\right)\right) .
$$

\section{The Chas-Sullivan algebra structure on $\mathbb{H}_{*}(L M)$ and its dual}

We assume in this section and in the following ones that $\boldsymbol{k}$ is a field of characteristic zero.

Denote by $p_{0}: L M \rightarrow M$ the evaluation map at the base point of $S^{1}$, and recall that the space $L M$ can be replaced by a smooth manifold ([4], [25]) so that $p_{0}$ is a smooth locally trivial fibre bundle ([1], [25]).

The Chas-Sullivan product

$$
\bullet: H_{*}(L M)^{\otimes 2} \longrightarrow H_{*-m}(L M), \quad x \otimes y \longmapsto x \bullet y
$$

was first defined in [3] by using "transversal geometric chains". Then

$$
\mathbb{H}_{*}(L M):=H_{*+m}(L M)
$$

becomes a commutative graded algebra.

It is convenient for our purpose to introduce the dual of the loop product $H^{*}(L M) \rightarrow H^{*+m}\left(L M^{\times 2}\right)$. Consider the commutative diagram

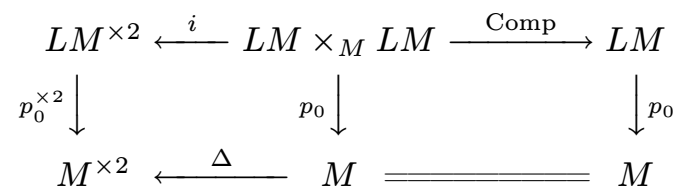

where

- Comp denotes composition of free loops,

- the left hand square is a pullback diagram of locally trivial fibrations,

- $i$ is the embedding of the manifold of composable loops into $L M \times L M$. 
The embeddings $\Delta$ and $i$ have both codimension $m$. Thus, using the ThomPontryagin construction we obtain the Gysin maps

$$
\Delta^{!}: H^{k}(M) \longrightarrow H^{k+m}\left(M^{\times 2}\right), \quad i^{!}: H^{k}\left(L M \times_{M} L M\right) \longrightarrow H^{k+m}\left(L M^{\times 2}\right) .
$$

Thus diagram (1) yields the diagram

$$
\begin{array}{ccc}
H^{k+m}\left(L M^{\times 2}\right) \stackrel{i^{!}}{\longleftarrow} H^{k}\left(L M \times_{M} L M\right) \stackrel{H^{k}(\mathrm{Comp})}{\longleftarrow} H^{k}(L M) \\
H^{*}\left(p_{0}\right) \uparrow & \uparrow H^{*}\left(p_{0}\right) \\
H^{*}\left(p_{0}\right)^{\otimes 2} \uparrow & H^{k}(M) \\
H^{k+m}\left(M^{\times 2}\right) \longleftarrow \Delta^{!} & H^{k}(M)=
\end{array}
$$

Following [27], [6], the dual of the loop product is defined by composition of maps on the upper line :

$$
i^{!} \circ H^{*}(\mathrm{Comp}): H^{*}(L M) \longrightarrow H^{*+m}\left(L M^{\times 2}\right) .
$$

\section{Proof of Proposition 1 and the Cohen-Jones-Yan spectral sequence.}

The composition of free loops Comp : $L M \times_{M} L M \rightarrow L M$ is obtained by pullback from the composition of paths Comp' ${ }^{\prime} M^{I} \times_{M} M^{I} \rightarrow M^{I}$ in the following commutative diagram.

(Comp)

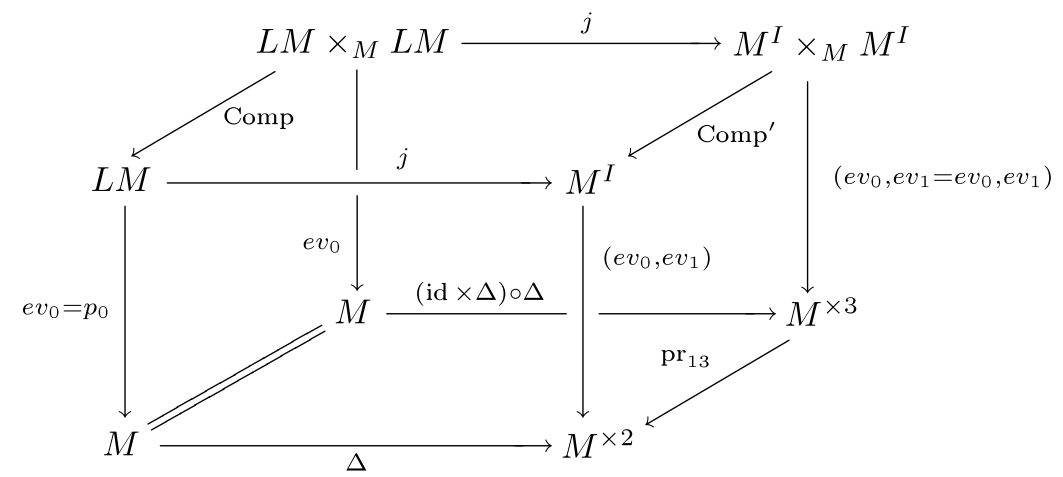

Here $\Delta$ denotes the diagonal embedding, $j$ the obvious inclusions, $e v_{t}$ denotes the evaluation maps at $t$, and $\operatorname{pr}_{13}$ the map defined by $\operatorname{pr}_{13}(a, b, c)=(a, c)$.

Let $(A, d)$ be a commutative differential graded algebra quasi-isomorphic to the differential graded algebra $C^{*}(M)$. A cochain model of the right hand square in diagram (Comp) is given by the commutative diagram

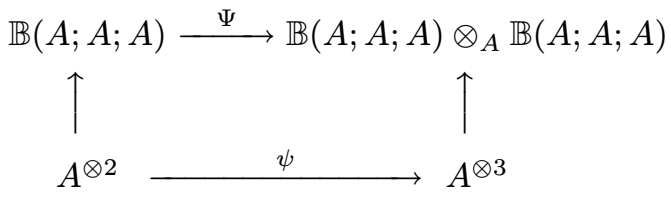

TOME $136-2008-\mathrm{N}^{\mathrm{O}} 2$ 
where $\Psi$ and $\psi$ denote the homorphism of cochain complexes defined by

$$
\begin{aligned}
\Psi\left(a \otimes\left[a_{1}|\cdots| a_{k}\right] \otimes a^{\prime}\right) & =\sum_{i=0}^{k} a \otimes\left[a_{1}|\cdots| a_{i}\right] \otimes 1 \otimes\left[a_{i+1}|\cdots| a_{k}\right] \otimes a^{\prime}, \\
\psi\left(a \otimes a^{\prime}\right) & =a \otimes 1 \otimes a^{\prime} .
\end{aligned}
$$

We consider now the commutative diagram obtained by tensoring diagram $(\dagger)$ by $A$ :

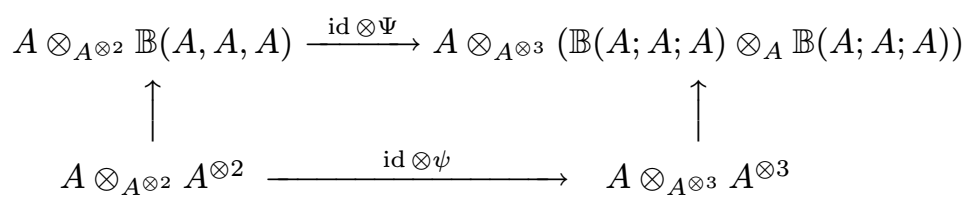

Since $\mathbb{B}(A ; A ; A)$ is a semifree model of $A$ as $A$-bimodule, we deduce from [8], p. 78, that diagram $(\ddagger)$ is a cochain model of the left hand square in diagram (Comp). Obviously, we have also the commutative diagram

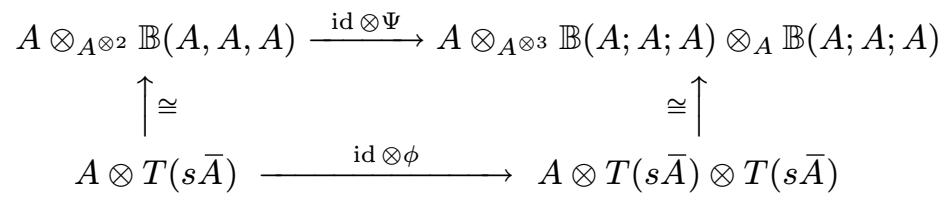

where $\phi$ denotes the coproduct of the coalgebra $T(s \bar{A})$. Thus we have proved:

Lemma 2. - The cochain complex $\boldsymbol{C}_{*}(A ; A)$ is a cochain model of LM, (i.e. we have an isomorphism of graded vector spaces $H H_{*}(A ; A) \cong H^{*}(L M)$.) Moreover, the composite

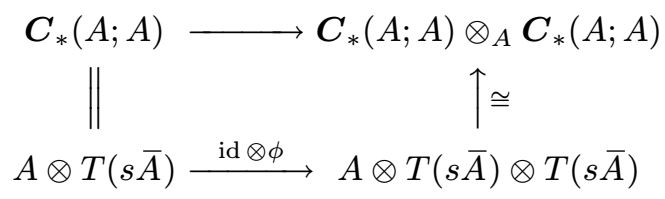

is model of the composition of free loops.

Recall now that the Gysin map $\Delta^{!}$of the diagonal embedding $\Delta: M \rightarrow$ $M \times M$ is the Poincaré dual of the homomorphism $H_{*}(\Delta)$. This means that the following diagram is commutative:

$$
\begin{gathered}
H_{*}(M) \stackrel{H_{*}(\Delta)}{\longrightarrow} H_{*}(M \times M) \\
-\cap[M] \uparrow \cong \\
H^{*}(M) \stackrel{\Delta^{!}}{\longrightarrow} H^{*}(M \times M[M \times M]
\end{gathered}
$$


Let $A$ be a Poincaré duality model of $M$ and $\mu_{A}$ as defined by diagram (2) of the introduction. The linear map $\mu_{A}=A \rightarrow A \otimes A$ is a cochain model for $\Delta$ ! Next observe that, [26], we can choose the pullback of a tubular neighborhood of the diagonal embedding $\Delta$ as a tubular neighborhood of the embedding $i: L M \times_{M} L M \rightarrow L M \times L M$. Thus the Gysin map $i^{!}$is obtained by pullback from $\Delta^{\text {! }}$. Therefore, since $A$ is graded commutative, then $C_{*}(A ; A)$ is a $A$ semifree and we have proved:

Lemma 3. - The linear map of degree $m$

$$
\boldsymbol{C}_{*}(A ; A) \otimes_{A} \boldsymbol{C}_{*}(A ; A) \stackrel{\cong}{\longrightarrow} A \otimes_{A^{\otimes 2}} \boldsymbol{C}_{*}(A ; A)^{\otimes 2} \stackrel{\mu_{A} \otimes \mathrm{id}}{\longrightarrow} \boldsymbol{C}_{*}(A ; A)^{\otimes 2}
$$

commutes with the differential and induces $i^{!}$in homology.

Then a combination of Lemmas 2, 3 and Lemma 4 below gives Proposition 1 of the introduction.

LEMMA 4. - The duality isomorphism $\left(H H_{*+m}(A ; A)\right)^{\vee} \cong H H^{*+m}\left(A ; A^{\vee}\right) \stackrel{(\theta)}{\cong}$ $H H^{*}(A ; A)$ transfers the map induced by $\Phi$ on $H_{*}(A ; A)$ to the Gerstenhaber product on $H H^{*}(A ; A)$.

Proof. - Observe that the composite (dotted arrow in the next diagram) induces the Gerstenhaber product in $H H^{*}(A ; A)$.

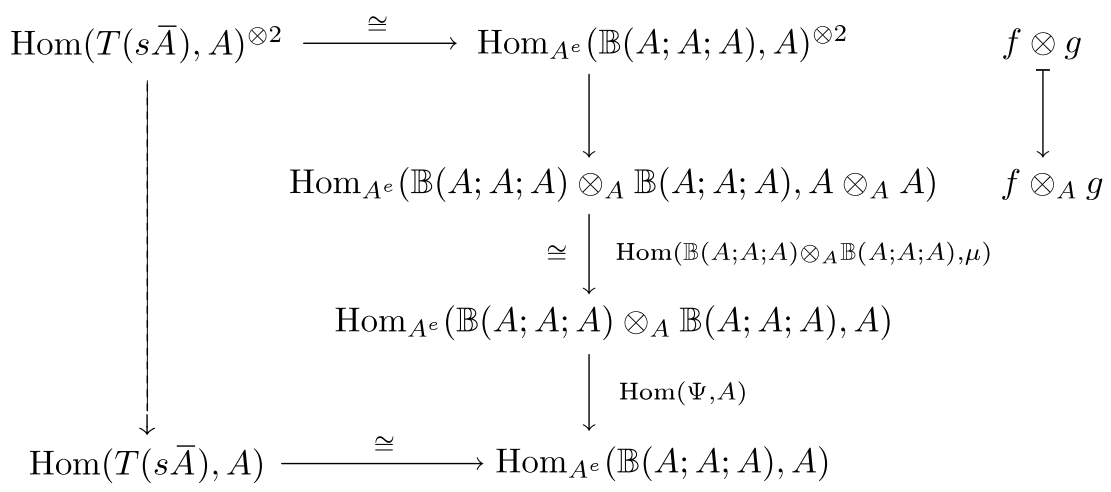

Then the remaining of the proof follows by considering an obvious commutative diagram.

томе $136-2008-\mathrm{N}^{\mathrm{O}} 2$ 
Spectral sequence. - By putting $F_{p}:=A \otimes(T(s \bar{A}))^{\leq p}$, for $p \geq 0$, we define a filtration

$$
A \otimes T(s \bar{A}) \supset \cdots \supset F_{p} \supset F_{p-1} \supset \cdots \supset A=F_{0}
$$

such that $\partial F_{p} \subset F_{p}$ and $\Phi\left(F_{p}\right) \subset \bigoplus_{k+\ell=p} F_{k} \otimes F_{\ell}$. The resulting spectral sequence

$$
E_{2}^{p, q}=H^{q}(M) \otimes H^{p}(\Omega M) \Longrightarrow H^{p+q}(L M)
$$

is the comultiplicative "regraded" Serre spectral sequence for the fibration $p_{0}$ : $L M \rightarrow M$. It dualizes into a spectral sequence of algebras

$$
H_{q+m}(M) \otimes H_{p}(\Omega M) \Longrightarrow \mathbb{H}_{p+q}(L M) .
$$

We recover in this way, for coefficients in a field of characteristic zero, the spectral sequence defined previously by Cohen, Jones and Yan [6].

\section{Proof of Proposition 2.}

Let $\rho: S^{1} \times L M \rightarrow L M$ be the canonical action of the circle on the space $L M$. The action $\rho$ induces an operator $\Delta: \mathbb{H}_{*}(L M) \rightarrow \mathbb{H}_{*+1}(L M)$. The Chas-Sullivan product together with $\Delta$ gives to $\mathbb{H}_{*}(L M)$ a BV-structure [3].

Denote by $\mathfrak{M}_{M}=(\bigwedge V, d)$ a (non necessary minimal) Sullivan model for $M$ $[8, \S 12]$. We put $s V=\bar{V}$ and denote by $S$ the derivation of $\wedge V \otimes \wedge \bar{V}$ defined by $S(v)=\bar{v}$ and $S(\bar{v})=0$ for $v \in V$ and $\bar{v} \in \bar{V}$. Then a Sullivan model for $L M$ is given by the commutative differential graded algebra $(\bigwedge V \otimes \wedge \bar{V}, \bar{d})$ where $\bar{d}(\bar{v})=-S(d v)$ [34]. Moreover in [33] Burghelea and Vigué prove that a Sullivan model of the action $\rho: S^{1} \times L M \rightarrow L M$ is given by

$$
\begin{gathered}
\left.\mathfrak{M}_{\rho}:(\bigwedge V \otimes \bigwedge \bar{V}, \bar{d}) \longrightarrow(\bigwedge u, 0) \otimes(\bigwedge V \otimes \bigwedge \bar{V}, \bar{d}), \quad|u|=1\right), \\
\mathfrak{M}_{\rho}(\alpha)=1 \otimes \alpha+u \otimes S(\alpha), \quad \alpha \in \bigwedge V \otimes \bigwedge \bar{V}
\end{gathered}
$$

In particular the map induced in cohomology by the action of $S^{1}$ on $L M$ is given by the derivation $S: H^{*}(\bigwedge V \otimes \bigwedge \bar{V}) \rightarrow H^{*-1}(\bigwedge V \otimes \bigwedge \bar{V})$. Denote now by $B$ the Connes' boundary on $C_{*}\left(\mathfrak{M}_{M} ; \mathfrak{M}_{M}\right)=\bigwedge V \otimes T(s \overline{\bigwedge V})$. D. Burghelea and M. Vigué proved the following lemma in [31, Thm. 2.4].

Lemma 5. - The morphism $f: \boldsymbol{C}_{*}\left(\mathfrak{M}_{M} ; \mathfrak{M}_{M}\right) \rightarrow\left(\mathfrak{M}_{M} \otimes \wedge \bar{V}\right)$ defined by

$$
f\left(a \otimes\left[a_{1}|\cdots| a_{n}\right]\right)=\frac{1}{n !} a S\left(a_{1}\right) \cdots S\left(a_{n}\right)
$$

is a quasi-isomorphism of complexes and $f \circ B=S \circ f$.

Lemma 5 identifies the Connes boundary, $B$ acting on $H H_{*}(A ; A) \cong$ $H_{*}\left(\mathfrak{M}_{M} ; \mathfrak{M}_{M}\right)$ with the circle action and thus with the Chas-Sullivan BVoperator on $H^{*}(L M) \cong H H_{*}(A ; A)$. This is Proposition 2 of the introduction. 


\section{Hodge decomposition}

With the notation of the previous sections, let $\left(\mathfrak{M}_{M} \otimes \bigwedge \bar{V}, \bar{d}\right)$ be a Sullivan model for $L M$. Denote by $G^{p}=\bigwedge V \otimes \bigwedge^{p} \bar{V}$ the subvector space generated by the words of length $p$ in $\bar{V}$. The differential $\bar{d}$ satisfies $\bar{d}\left(G^{p}\right) \subset G^{p}$. Thus we put

$$
H_{[p]}^{n}(L M):=H^{n}\left(G^{p}\right) .
$$

This decomposition splits $H^{*}(L M ; \boldsymbol{k})$ into summands given as eigenspaces of the maps $L M \rightarrow L M$ induced from the $n$-power maps of the circle $e^{i t} \mapsto e^{i n t}$ [33]. It defines by duality a Hodge decomposition on $H_{*}(L M)$. We are now ready to prove Theorem 2 of the introduction.

Proof of Theorem 2. - Recall that the differential $\partial$ in $C^{*}\left(\mathfrak{M}_{M} ; \mathfrak{M}_{M}\right)$ decomposes into $\partial=\partial_{0}+\partial_{1}$ with $\partial_{0}\left(\mathfrak{M}_{M} \otimes T^{p}(s \overline{\wedge V})\right) \subset \mathfrak{M}_{M} \otimes T^{p}(s \overline{\wedge V})$, and $\partial_{1}\left(\mathfrak{M}_{M} \otimes T^{p}(s \overline{\wedge V})\right) \subset \mathfrak{M}_{M} \otimes T^{p-1}(s \overline{\wedge V})$.

We consider the quasi-isomorphism $f: C^{*}\left(\mathfrak{M}_{M} ; \mathfrak{M}_{M}\right) \rightarrow\left(\mathfrak{M}_{M} \otimes \wedge \bar{V}, \bar{d}\right)$ defined in Lemma 5. If we apply Lemma 5 , when $d=0$ in $\wedge V$, we deduce that Ker $f$ is $\partial_{1}$-acyclic.

Lemma 6. - Let us define $K^{(p)}:=\operatorname{Ker} f \cap\left(\mathfrak{M}_{M} \otimes T^{p}(s \overline{\wedge V})\right)$.

1) If $\omega \in K^{(p)} \cap$ Ker $\partial$ then there exists $\omega^{\prime} \in \bigoplus_{r \geq p+1} K^{(r)}$ such that $\partial \omega^{\prime}=\omega$.

2) $f$ induces a surjective map

$$
\left(\mathfrak{M}_{M} \otimes T^{\geq p}(s \overline{\wedge V})\right) \cap \operatorname{Ker} \partial \longrightarrow\left(\mathfrak{M}_{M} \otimes \wedge^{p} s V\right) \cap \operatorname{Ker} \bar{d} .
$$

Proof. - If $\omega \in K^{(p)} \cap$ Ker $\partial$ then $\omega=\partial(u+v)$ with $u \in K^{(p)}$ and $v \in K^{(\geq p+1)}$. Since $\partial_{1} u=0$ we have $u=\partial \beta_{1}$ some $\beta \in K^{(p+1)}$ and thus $\omega-d \beta_{1} \in K^{(\geq p+1)}$. An induction on $n \geq 1$ we prove that there exists $\beta_{n} \in K^{(p+n)}$ such that $\omega-d \beta_{n} \in K^{(p+n)}$. Since $\bigwedge V$ is 1-connected $\left(\mathfrak{M}_{M} \otimes T^{p+n}(s \overline{\wedge V})\right)^{|\omega|}=0$ for some integer $n_{0}$. We put $\omega^{\prime}=\beta_{n_{0}}$.

In order to prove the second statement, we consider a $\bar{d}$-cocycle $\alpha \in \mathfrak{M}_{M} \otimes$ $\bigwedge^{p} s V$ and we write $\alpha=f(\omega)$ for some $\omega \in \mathfrak{M}_{M} \otimes T^{p}(s \overline{\wedge V})$. It follows from the definition of $f$ that $\partial \omega \in K^{(p-1)}$. Thus, by the first statement, $\partial \omega=\partial \omega^{\prime}$ some $\omega^{\prime} \in K^{(\geq p)}$. Then $\varpi=\omega-\omega^{\prime}$ is $\partial$-cocycle of $K^{\geq p}$ such that $f(\varpi)=\alpha$.

To end the proof of Theorem 2, let us consider $\alpha \in H_{[n]}^{*}(L M)$. By Lemma 6, $\alpha$ is the class of $f(\beta)$ where $\beta \in \mathfrak{M}_{M} \otimes T^{\geq n}(s \overline{\bigwedge V})$. Therefore $\Phi(\beta)$ belongs to $\bigoplus_{i+j>n}\left(\mathfrak{M}_{M} \otimes T^{i}(s \overline{\wedge V})\right) \otimes\left(\mathfrak{M}_{M} \otimes T^{j}(s \overline{\wedge V})\right)$ (see Lemma 2). Now since $f\left(\mathfrak{M}_{M} \otimes T^{p}(s \overline{\wedge V})\right) \subset \mathfrak{M}_{M} \otimes \bigwedge^{p} s V$,

$$
[\Phi(\alpha)] \in \bigoplus_{i+j \geq n} H_{[i]}^{*}(L M) \otimes H_{[j]}^{*}(L M) .
$$

TOME $136-2008-\mathrm{N}^{\mathrm{O}} 2$ 
Now, as announced in the introduction (Proposition 3) there is an other interpretation of $H_{[p]}^{n}(L M)$ in terms of the cohomology of a differential graded Lie algebra.

Let $L$ be a differential graded algebra $L$ such that the cochain algebra $\mathcal{C}^{*}(L)$ is a Sullivan model of $M,[8$, p. 322]. In particular, the homology of the enveloping universal algebra of $L$, denoted $U L$, is a Hopf algebra isomorphic to $H_{*}(\Omega M)$. We consider the cochain complex $\mathcal{C}^{*}\left(L ; U L_{a}^{\vee}\right)$ of $L$ with coefficients in $U L^{\vee}$ considered as an $L$-module for the adjoint representation. We have shown (see [12, Lemma 4]) that the natural inclusion $\mathcal{C}^{*}(L) \hookrightarrow \mathcal{C}^{*}\left(L ; U L_{a}^{\vee}\right)$ is a relative Sullivan model of the fibration $p_{0}: L M \rightarrow M$. Write $\mathcal{C}^{*}(L)=(\bigwedge V, d)$, then $V=(s L)^{\vee}$ and $\bar{V}=L^{\vee}$. There is also (Poincaré-Birkoff-Witt Theorem) an isomorphism of graded coalgebras, [8, Prop. 21.2]:

$$
\gamma: \wedge L \longrightarrow U L, \quad x_{1} \wedge \cdots \wedge x_{k} \longmapsto \sum_{\sigma \in \mathfrak{S}_{k}} \epsilon_{\sigma} x_{\sigma(1)} \cdots x_{\sigma(k)} .
$$

If we put $\Gamma^{p}=\gamma\left(\bigwedge^{p} V\right)$ we obtain the following isomorphisms of cochain complexes

$$
(\bigwedge V \otimes \bigwedge \bar{V}, \bar{d}) \cong \mathcal{C}^{*}\left(L ; U L_{a}^{\vee}\right), \quad G^{p} \cong \mathcal{C}^{*}\left(L ;\left(\Gamma^{p}\right)^{\vee}\right)
$$

which in turn induce the isomorphisms

$$
\mathbb{H}^{*}(L M) \cong \operatorname{Ext}_{U L}\left(\boldsymbol{k}, U L_{a}^{\vee}\right), \quad \mathbb{H}_{[p]}^{*}(L M) \cong \operatorname{Ext}_{U L}\left(\boldsymbol{k}, \Gamma^{p}(L)^{\vee}\right)
$$

and by duality,

$$
\mathbb{H}_{*}(L M) \cong \operatorname{Tor}^{U L}\left(\boldsymbol{k}, U L_{a}\right), \quad \mathbb{H}_{*}^{[p]}(L M) \cong \operatorname{Tor}^{U L}\left(\boldsymbol{k}, \Gamma^{p}\right) .
$$

\section{BIBLIOGRAPHY}

[1] J.-L. BRYLINSKI - Loop spaces, characteristic classes and geometric quantization, Progress in Mathematics, vol. 107, Birkhäuser, 1993.

[2] D. Burghelea \& M. Vigué-Poirrier - "Cyclic homology of commutative algebras I", Proceedings of the Meeting on Algebraic Homotopy, Louvain, 1986, Lectures Notes in Math. 1318 (1988), p. 51-72.

[3] M. Chas \& D. Sullivan - "String topology", preprint arXiv:math.GT/9911159, 1999.

[4] D. Chataur - "A bordism approach to string topology", Int. Math. Res. Not. 46 (2005), p. 2829-2875.

[5] R. L. Cohen \& J. D. S. Jones - "A homotopy theoretic realization of string topology", Math. Ann. 324 (2002), p. 773-798.

[6] R. L. Cohen, J. D. S. Jones \& J. YAN - The loop homology algebra of spheres and projective spaces, Progr. Math., vol. 215, Birkhäuser, 2004. 
[7] Y. FÉlix, S. Halperin \& J.-C. Thomas - Differential graded algebras in topology, North-Holland, 1995.

[8] _ Rational homotopy theory, Graduate Texts in Mathematics, vol. 205, Springer, 2001.

[9] Y. Félix, L. Menichi \& J.-C. Thomas - "Gerstenhaber duality in Hochschild cohomology", J. Pure Appl. Algebra 199 (2005), p. 43-59.

[10] Y. FÉlix \& J.-C. Thomas - "Monoid of self-equivalences and free loop spaces", Proc. Amer. Math. Soc. 132 (2004), p. 305-312.

[11] Y. Felix, J.-C. Thomas \& M. Vigué-Poirrier - "The Hochschild cohomology of a closed manifold", Publ. Math. Inst. Hautes Études Sci. 99 (2004), p. 235-252.

[12] Y. Félix, J.-C. Thomas \& M. Vigué-Poirrier - "Rational string topology", J. Eur. Math. Soc. (JEMS) 9 (2007), p. 123-156.

[13] K. FUJII - "Iterated integrals and the loop product", preprint arXiv:math/07040014.

[14] M. Gerstenhaber - "The cohomology structure of an associative ring", Ann. of Math. (2) 78 (1963), p. 267-288.

[15] M. Gerstenhaber \& S. D. SchaK - "A Hogde type decomposition for commutative algebras", J. Pure Appl. Algebra 48 (1987), p. 229-289.

[16] V. GinsburG - "Calabi-Yau algebras", preprint arXiv:math/0612139.

[17] K. Gruher \& P. SAlvatore - "Generalized string topology operations", preprint arXiv:math.AT/0602210.

[18] A. Hamilton \& A. LAZAREV - "Homotopy algebras and noncommutative geometry", preprint arXiv:math.QA/0410621.

[19] J. D. S. Jones - "Cyclic homology and equivariant homology", Invent. Math. 87 (1987), p. 403-423.

[20] P. Lambrechts \& D. Stanley - "Poincaré duality and commutative differential graded algebras", preprint arXiv:math/0701309.

[21] J.-L. LODAY - "Opérations sur l'homologie cyclique des algèbres commutatives", Invent. Math. 96 (1989), p. 205-230.

[22] L. MENICHI - "String topology for spheres", preprint arXiv:math/AT/0609304.

[23] _ "Batalin-Vilkovisky algebras and cyclic cohomology of Hopf algebras", K-Theory 32 (2004), p. 231-251.

[24] S. A. Merkulov - "De Rham model for string topology", Int. Math. Res. Not. (2004), p. 2955-2981.

[25] A. Stacey - "The differential topology of loop spaces", preprint arXiv:math.DG/0510097.

[26] J. STASHEFF - "The intrinsic bracket on the deformation complex of an associative algebra", J. Pure Appl. Algebra 89 (1993), p. 231-235.

TOME $136-2008-\mathrm{N}^{\mathrm{O}} 2$ 
[27] D. Sullivan - "Open and closed string field theory interpreted in classical algebraic topology", in Topology, geometry and quantum field theory, London Math. Soc. Lecture Note Ser., vol. 308, Cambridge Univ. Press, 2004, p. 344-357.

[28] T. TRADLER - "The BV algebra on Hochschild cohomology induced by infinity inner products", preprint arXiv:math.QA/0210150.

[29] T. Tradler \& M. Zeinalian - "Infinity structure of Poincaré duality spaces", Algebr. Geom. Topol. 7 (2007), p. 233-260, Appendix by Dennis Sullivan.

[30] D. VAintrob - "The string topology BV algebra, Hochschild cohomology and the Goldman bracket on surfaces", preprint arXiv:math/0702859.

[31] M. Vigué-Poirrier - "Homologie de Hochschild et homologie cyclique des algèbres différentielles graduées", Astérisque 191 (1990), p. 7, 255-267, International Conference on Homotopy Theory (Marseille-Luminy, 1988).

[32] _ "Décompositions de l'homologie cyclique des algèbres différentielles graduées commutatives", K-Theory 4 (1991), p. 399-410.

[33] M. Vigué-Poirrier \& D. BurgheleA - "A model for cyclic homology and algebraic $K$-theory of 1 -connected topological spaces", J. Differential Geom. 22 (1985), p. 243-253.

[34] M. Vigué-Poirrier \& D. Sullivan - "The homology theory of the closed geodesic problem", J. Differential Geometry 11 (1976), p. 633-644. 